\title{
Design Instrucional: um comparativo de metodologias para definição de abordagem em mundo virtual
}

\author{
Andressa Falcade ${ }^{1}$, Aliane L. Krassmann ${ }^{1}$, Vania Freitas ${ }^{1}$, Tassiana Kautzmann ${ }^{1}$, \\ Rafaela R. Jardim ${ }^{1}$, Giliane Bernardi ${ }^{1}$, Roseclea D. Medina ${ }^{1}$ \\ ${ }^{1}$ PPGI - Universidade Federal de Santa Maria (UFSM) \\ \{andressafalcade, alkrassmann, 2.vania, tassik, rjardim, bgiliane, \\ roseclea.medina\} @gmail.com,
}

\begin{abstract}
Instructional methods are processes that assist on organizing and structuring courses in the educational field. This study analyzed eight instructional methods in order to choose the most suitable for the construction of an Instructional Design for Computer Networks teaching within the Virtual World $(V W)$ TCN5. The phases of each method were separated according to design and implementation stages of Classic Instructional Design. At the end of the study, ADDIE ASSURE and Dick and Carey methods were the most suited to the research scope.
\end{abstract}

Resumo. Métodos instrucionais são processos que auxiliam na organização e estruturação de cursos no campo da educação. Neste estudo foram analisados oito métodos instrucionais, a fim de eleger o mais adequado para a construção de um Design Instrucional para o ensino de Redes de Computadores dentro do Mundo Virtual (MV) TCN5. As fases de cada método foram separadas de acordo com as etapas de concepção e execução do Design Instrucional Clássico. Ao final do estudo os métodos ADDIE e Dick e Carey foram os que mais se adequaram ao escopo da pesquisa.

\section{Introdução}

A utilização de Mundos Virtuais (MV) no âmbito educativo está se tornando mais frequente, tanto na educação presencial como à distância. Segundo Holden et al. (2010) os MV facilitam o ensino centrado no aluno, permitindo um aprendizado mais significativo. Apesar disso, Nascimento (2006) destaca que apenas a existência da tecnologia no meio escolar não garante o aprendizado do estudante. Nesse sentido, o Design Instrucional (DI) surge como uma oportunidade de gerar maior aproveitamento dos recursos educacionais disponíveis para a educação.

Segundo Branch (2009), o DI é um processo iterativo para o planejamento de objetivos de desempenho, seleção de estratégias instrucionais, escolha de mídias, seleção e criação de materiais, e avaliação. É construído tendo métodos instrucionais existentes como norteadores, que permitem às pessoas a visualização de todo $o$ processo, estabelecendo diretrizes para o gerenciamento de cursos.

Este estudo tem por objetivo comparar nove metodologias instrucionais (ADDIE, ASSURE, Gerlac e Ely, PIE, AIM-CID, DEI, 4C/ID, ILDF on-line e Dick e Carey) no intuito de apresentar características, aplicações, vantagens e limitações, destacando diferenças, semelhanças e enfoques pedagógicos de cada método. A pesquisa motivou-se da necessidade de escolha do método que mais se adequasse à construção de um Design Instrucional para a aprendizagem dentro do MV TCN5 [Voss 2014], que aborda o tema Rede de Computadores com ambientes adaptados às características de contexto: estilo cognitivo e nível de conhecimento do aluno. 
Um Mundo Virtual é uma simulação de um ambiente real, ou a formulação de um ambiente imaginário, fictício, criado para convivência e comunicação entre pessoas representadas por avatares que realizam ações e interagem entre si [Backes 2012]. Os autores Mueller et al. (2011) especificam melhor o que são MV, afirmando que: devem permitir a interação síncrona entre muitos usuários, mesmo eles não estando no mesmo espaço físico; devem existir diversas formas de comunicação, como texto, voz e linguagem corporal; devem ser persistentes (continuar funcionando, mesmo que não estejam sendo usados); e devem lembrar a localização das pessoas, a posse dos objetos e, principalmente, com avatares que representem os usuários.

\section{Design Instrucional e educação: métodos de desenvolvimento}

A utilização da tecnologia no ambiente educativo vem sendo cada vez mais estudada, no intuito de melhorar e aperfeiçoar as formas de aprendizagem. Estudos que expõem ferramentas tecnológicas educativas já estão apresentando maneiras de garantir o melhor roteiro pedagógico para a geração do conhecimento.

A organização dos recursos pedagógicos e dos conteúdos a serem estudados pode influenciar na forma com que o estudante assimilará determinado saber, podendo essa aprendizagem ocorrer mais facilmente quando essa organização ocorre de acordo com as características do aluno (e.g. estilos cognitivos, nível de conhecimento, preferências, localização, tipo de dispositivo, velocidade de rede e tamanho de tela).

Para que seja possível determinar um arranjo adequado para cada estudante, de acordo com suas preferências, existem algumas metodologias que permitem um planejamento minucioso, com etapas pré-programadas, podendo ser citadas as Arquiteturas Pedagógicas (AP), o Design Instrucional (DI) e o Planejamento Instrucional (PI). No âmbito deste estudo, o termo utilizado faz referência ao DI, por apresentar maior afinidade com o uso de tecnologias e recursos metodológicos e informacionais em termos de abrangência mundial.

DI é uma ação que abrange planejamento, desenvolvimento e aplicação de técnicas, no intuito de gerar a aprendizagem humana [Filatro 2008], apresentando objetivos orientados e focados no desempenho significativo [Branch 2009]. Para Heidrich et al. (2007), o DI adequado faz o estudante aprender o seu máximo da melhor forma, permitindo a relação entre educação, comunicação e tecnologia, a fim de obter maior nível de atenção para a aprendizagem de novos saberes.

Conforme Behar et al. (2008) o termo design constitui projetar, compor visualmente ou colocar em prática um plano intencional que, segundo Filatro (2008), deve conter técnicas e métodos aplicados em situações educacionais específicas, com o intuito de gerar aprendizagem. Já instrução é a atividade de ensino que se utiliza da comunicação para facilitar a aprendizagem [Filatro 2008]. Modelos de projeto instrucional que auxiliam na construção e estruturação de cursos são representações que indicam o ponto de vista de como as pessoas aprendem, e podem ser utilizados como guias na construção de um DI [Herridge 2015]. Para desenvolver um projeto instrucional existem alguns métodos que descrevem a organização, o particionamento e a condução do aprendizado [Vahldick e Knaul 2010], dentre eles estão o ADDIE, ASSURE, Gerlach e Ely, PIE, AIM-CID, DEI, 4C/ID, ILDF Online e Dick e Carey.

Nesta pesquisa esses modelos serão avaliados de acordo com as necessidades de aplicação no Mundo Virtual TCN5 no intuito de eleger àquele que será utilizado como base para a estruturação do curso de Redes de Computadores para o ambiente virtual imersivo. Serão consideradas como parâmetro as duas fases do Design Instrucional 
V Congresso Brasileiro de Informática na Educação (CBIE 2016)

Anais do XXVII Simpósio Brasileiro de Informática na Educação (SBIE 2016)

Clássico de Filatro (2008): concepção, que envolve todo o planejamento do curso a ser desenvolvido; e execução, que aborda a implementação e utilização do curso.

\subsection{ADDIE}

O ADDIE foi definido por Clark (2014) como um processo sistemático e iterativo que serve para o desenvolvimento de experiências de aprendizagem, com o intuito de aperfeiçoar capacidades e saberes. Segundo o autor, ele foi baseado nas fases do método System Design Instrucional (ISD), utilizado no departamento de defesa dos Estados Unidos na década de 1970, sendo o seu nome definido pelas iniciais de cada etapa do processo: Analysis, Design, Development, Implementation e Evaluation [Clark 2014].

Tendo fases bem definidas, o modelo se desenvolve de forma iterativa, podendo ser adaptado às especificidades de cada curso, professor e estudante. É utilizado principalmente em ambientes educacionais para facilitar a construção de conhecimentos e competências em episódios de aprendizagem guiada [Branch 2009].

\subsection{ASSURE}

O método ASSURE foi desenvolvido com o objetivo de relatar tarefas para a seleção e utilização de mídias instrucionais [Santos 2010]. Tem como características perspectivas construtivistas e a possibilidade de integração multimídia [Mallmann 2008].

Apresenta seis tarefas que compõem o seu nome: analyze learners; state objectives; select media and materials; utilize media and; require learner participation; e evaluate and revise [Santos 2010]. Bandeira (2009) destaca que o modelo sugere a sequência de um roteiro de planejamento e direção instrucional para utilizar mídias.

\subsection{Gerlach e Ely}

De acordo com Grabowski (2003), o modelo Gerlach e Ely foi criado a partir da necessidade de uma visão abrangente do ensino e aprendizagem. Segundo Mallmann (2008), é um modelo prescritivo e situado na educação superior.

O Gerlach e Ely é uma tentativa de retratar graficamente um método de planejamento sistemático de instruções, incorporando dois itens: a necessidade de objetivos cuidadosamente definidos $\mathrm{e}$ as táticas sobre como atingir cada meta (Grabowski, 2003). Apresenta o roteiro para um bom ensino e aprendizagem e também serve como um lembrete para os componentes importantes [Grabowski 2003].

\subsection{Newby, Stepich, Lehman e Russell Instructional Model (PIE)}

O PIE é aplicado pelos professores para renovar métodos de ensino em sala de aula e viabilizar uma maior aprendizagem entre alunos por meio da utilização de mídias, sendo composto das fases: planejamento, implantação e avaliação [Gutierrez 2013].

Segundo Gutierrez (2013) a fase de planejamento define como as aulas serão apresentadas; na fase de implementação trabalham-se métodos de ensino-aprendizagem e meios de comunicação; e na fase de avaliação é verificada a aprendizagem do aluno e os métodos de ensino apresentados.

\subsection{Abordagem Integrada de Modelagem - Conceitual, Instrucional e Didática}

O IAM-CID (Abordagem Integrada de Modelagem - Conceitual, Instrucional e Didática) busca reunir em apenas uma proposta, as perspectivas de modelagem conceitual, instrucional e didática [Barbosa 2004]. A técnica de mapas conceituais é utilizada para exibir conceitos de hierarquia, facilitar sua utilização e ser aceito por profissionais de educação e outros modelos de conteúdos educacionais [Aceituno 2013].

$\mathrm{Na}$ etapa de modelagem conceitual define-se o nível de conhecimento do estudante para o ensino aprendizagem; na etapa instrucional, além de conceitos, podem 
ser inseridos elementos e informações para o conhecimento e domínio do assunto; já na etapa de modelagem didática toma-se como ponto de partida o modelo instrucional, apresentando estratégias de utilização e explanação do conteúdo, permitindo que seja adaptado para contextos diferentes [Barbosa 2004].

\subsection{Designing Effective Instruction (DEI)}

O modelo DEI tem como objetivo a flexibilidade do designer na escolha dos elementos que julga necessário utilizar, onde a perspectiva do aluno é considerada para a construção da instrução [Morrinson et al. 2004].

É composto por nove elementos chaves, que são independentes e flexíveis: 1. Identificar problemas instrucionais; 2. Examinar características dos alunos; 3. Identificar o conteúdo e analisar componentes de tarefas; 4. Especificar os objetivos instrucionais; 5. Sequenciar conteúdos dentro de cada unidade de ensino; 6. Projetar estratégias de ensino; 7. Planejar e desenvolvera instrução; 8. Desenvolver instrumentos de avaliação; 9. Selecionar os recursos de apoio às atividades [Alvarado et al. 2012].

\subsection{Four Component Instructional Design}

O modelo Four Component Instructional Design (4C/ID) sugere uma metodologia de aprendizagem prática, baseada em exercícios, que contém material de apoio para que o aluno consiga resolvê-los progredindo até solucioná-los sozinho [Vahldick 2007].

Segundo Merriënboer et al. (2002) o modelo é centrado na integração e atuação coordenada de habilidades constituintes de tarefas específicas, fazendo uma distinção fundamental entre a informação de apoio necessária e o desempenho exigido. Compõese de quatro fases: ativação, demonstração, aplicação e integração, e necessita da divisão do assunto em partes para que seja aplicado [Merriënboer et al. 2002].

\subsection{Integrative Lerning Design Framework (ILDF On-line)}

O modelo ILDF on-line é uma adaptação construtivista do modelo ADDIE, mais flexível e integrado à estrutura institucional para um curso online. Esse modelo contém três fases: exploração, enactment e avaliação (Araújo, 2009).

O modelo incorpora três componentes-chaves da aprendizagem online: 1. Os modelos pedagógicos, nos quais as visões do ensino e da aprendizagem representam modelos cognitivos; 2. As estratégias instrucionais, ou seja, aquilo facilita a aprendizagem do estudante; 3 . As tecnologias instrucionais, que se referem à descrição de várias tecnologias de aprendizagem. [Horvitz 2005].

\subsection{Dick e Carey}

O modelo Dick \& Carey foi desenvolvido em 1978 por Walter Dick, Lou e James Carey, sendo composto por dez fases de desenvolvimento [Clark 2004]. Esse modelo reflete que existe uma ligação previsível e fiável entre um estímulo (materiais de instruções) e a resposta que este produz em um aluno (aprendizagem dos materiais). As partes do sistema dependem umas das outras para a entrada e saída, e todo o sistema utiliza o feedback para determinar se o seu objetivo foi alcançado [Dick e Carey 1990].

McGriff (2001) aponta que algumas vantagens desse modelo são a aplicação em variados contextos e o ajuste às mudanças de teoria e prática. Como desvantagem o autor apresenta a aprendizagem não linear e a pressuposição de que a aprendizagem é previsível e confiável.

\section{Trabalhos Correlatos}

A fim de apresentar a relevância desta pesquisa, apresentam-se alguns trabalhos que corroboram com o estudo, pois compararam métodos e metodologias instrucionais. 
O trabalho de Mallmann (2008) realiza uma comparação de 16 modelos de designs instrucionais, buscando analisar dados como: autores, principais características, etapas e implicações docentes, sintetizando conceitualmente o design instrucional. Além disso, exibe as etapas e desdobramentos encontrados em todos os modelos, apresentando aspectos que os diferenciam, como: público alvo, soluções, estratégias e materiais, demonstrando que o professor e o design instrucional devem estar relacionados no processo de ensino e aprendizagem.

Já no estudo de Gutierrez (2013) é feita uma comparação entre os modelos de DI ADDIE e PIE. O autor monta uma tabela apresentando as fases de cada um dos modelos, fazendo uma comparação entre os aspectos em comum e as tarefas correspondentes. É destacado que o PIE volta-se para o ambiente de sala de aula, onde o professor define a estrutura do desenvolvimento, enquanto o ADDIE é mais estruturado e pode ser utilizado em ambientes diversificados. Finalizando o estudo, o autor chegou à conclusão de que o modelo PIE se mostrou mais simples, por permitir que o professor escolha o momento do DI que mais se adequa ao seu conteúdo.

O comparativo entre metodologias instrucionais realizada neste estudo é diferente das descritas anteriormente, pois analisa as fases de cada método, separando-as de acordo com as etapas concepção e execução do DI clássico, abordadas por Filatro (2008). Essa análise permitirá expor os métodos instrucionais que possuem mais recursos para aplicações educacionais, a fim de criar um DI dentro do MV TCN5.

\section{Método da Pesquisa}

Esta pesquisa tem enfoque não experimental [Wazlawick 2009], visando realizar uma análise comparativo-qualitativa das metodologias ADDIE, ASSURE, Gerlach e Ely, PIE, AIM-CID, DEI, 4C/ID, ILDF Online e Dick e Carey. Dessa forma tomou-se como base o contexto onde será desenvolvido o Design Instrucional para definir alguns critérios que deverão ser observados. O contexto de aplicação refere-se ao MV TCN5, que possui ambientes adaptados ao estilo cognitivo e nível de expertise do estudante.

Nesse sentido, os métodos serão analisados de acordo com a literatura existente, de forma a obter uma visão ampla de cada aplicação, suas vantagens e desvantagens para as características deste estudo. As etapas de cada método serão relacionadas às situações de aplicação do Design Instrucional Clássico, [FILATRO 2008], concepção e execução, para posterior checagem de pontos comuns em cada fase de aplicação, bem como os pontos diferenciais. A última parte da pesquisa se resume à apresentação dos resultados e a definição dos métodos que mais se adequaram ao escopo aqui explorado.

\section{Análise Comparativa e Resultados}

Esta seção apresenta uma descrição mais detalhada de cada método instrucional abordado neste estudo. Para tanto, as fases são dividas de acordo as etapas de execução e concepção do DI clássico [Filatro 2008]. São considerados nove métodos de desenvolvimento instrucional: ADDIE, AS-SURE, PIE, AIM-CID, 4C/ID, ILDF online, DEI, Gerlach e Ely e Dick e Carey.

$\mathrm{Na}$ etapa de concepção as metodologias ADDIE e ASSURE trazem a sua primeira fase: análise, que permite a observação das características do estudante (e.g. expertise, dispositivo, estilo cognitivo, experiência online, largura de banda, problemas emocionais, culturais e econômicos que podem influenciar no processo de aprendizagem) [Santos 2010]. Nos modelos AIM-CID, 4C/ID e Dick e Carey, no que diz respeito ao contexto do estudante, apenas o nível de conhecimento é definido. No 
V Congresso Brasileiro de Informática na Educação (CBIE 2016)

Anais do XXVII Simpósio Brasileiro de Informática na Educação (SBIE 2016)

modelo DEI, são examinadas características gerais que podem influenciar decisões na instrução. Já no modelo de Gerlach e Ely, características como conhecimentos prévios, atitudes e habilidades do estudante são vistos apenas na segunda fase. Nos modelos ILDF Online e PIE, não foram identificadas análises das características do estudante.

Os modelos ASSURE, Gerlach e Ely, DEI e Dick e Carey possuem uma fase específica para a definição dos objetivos da aprendizagem. Com relação à definição de conteúdos, conhecimentos, materiais, recursos e tecnologias, todos os modelos possuem uma fase que aborda essa seleção. No caso do ASSURE existe a fase de seleção de mídias e materiais, onde o professor faz a seleção dos que considera mais adequado para o ensino de determinado conteúdo. No PIE, essa seleção acontece na fase de implementação, enquanto que no AIM-CID é realizado no modelo didático.

No ADDIE as fases de design e desenvolvimento exploram a construção de um programa detalhado, a criação e a adaptação de materiais e recursos para utilização no curso e o desenvolvimento de itens de avaliação [Nunes e Schiel 2011]. O modelo Dick e Carey possui mais fases na etapa de concepção do modelo clássico, com sete das suas dez etapas voltadas ao planejamento. Diferencia-se dos demais por apresentar uma preocupação maior com o processo educativo, dando atenção especial ao roteiro a ser desenvolvido pelo estudante e aos resultados esperados [Dick e Carey 1990].

A etapa de execução definida por Filatro (2008) abrange as fases de: implementação e avaliação (ADDIE); utilização de mídias e materiais, requisição do aprendiz e avaliação e revisão (ASSURE); implementação e avaliação (PIE); avaliação (ILDF On-line); desenvolvimento e avaliação (DEI); desenvolvimento e avaliação de desempenho (Gerlach e Ely); e pré-avaliação, avaliação contínua e revisão do DI (Dick e Carey). Nas fases mencionadas os pontos em comum são a utilização do curso estruturado e a avaliação de desempenho do estudante e do DI. O modelo Gerlach e Ely, apresenta cinco atividades na fase de desenvolvimento, sendo elas: a definição de estratégias de ensino; a organização de grupos de estudantes e alocação de tempo de acordo com as estratégias; alocação de espaço; e seleção de recursos [Grabowski 2003].

A fase de avaliação no modelo ADDIE é dividida em duas partes: formativa e somativa. A primeira está presente em cada etapa do processo e a segunda consiste de testes projetados para domínios específicos e itens de critério de referência que oferecem oportunidades para o feedback dos usuários. No modelo Gerlach e Ely, a avaliação acontece quando o professor mede as realizações dos estudantes e suas atitudes em relação ao conteúdo e à instrução. No DEI é realizada uma avaliação dos objetivos instrucionais utilizados, enquanto que no modelo ASSURE é verificada a utilização de algumas mídias e seus resultados. No modelo AIM-CID existe um elemento de avaliação à parte das fases, que avalia o aprendiz e o conhecimento adquirido por ele [Aceituno 2013]. A fase de execução no modelo Dick e Carey é voltada totalmente à avaliação do DI modelado, resultando em sua última fase na revisão do DI a partir dos resultados obtidos [Clark 2004]. A partir das descrições anteriores, a Tabela 1 apresenta a divisão das fases dos modelos de acordo com as etapas de concepção e execução definidas por Filatro (2008). Nessa tabela pode ser visualizado que há uma diferenciação entre o número de fases de cada DI.

O modelo ILDF On-line e o modelo PIE apresentam somente três etapas em comparação com os demais métodos. Devido a essa diferenciação podem ser considerados menos completos, pois nas fases de exploração e planejamento, esses modelos não abrangem contexto tecnológico e do usuário nem a adaptação de materiais. 
V Congresso Brasileiro de Informática na Educação (CBIE 2016)

Anais do XXVII Simpósio Brasileiro de Informática na Educação (SBIE 2016)

Já o modelo Dick e Carey se destaca pela grande quantidade de fases na etapa de execução, mostrando seu detalhamento no planejamento do curso. Nas suas sete fases o professor: define os objetivos a serem alcançados; especifica os passos que o aluno deve ser capaz de executar para realizar as tarefas; identifica o nível de conhecimento dos estudantes; realiza uma descrição das tarefas e habilidades que serão aprendidas, além das condições, normas e critérios para execução de cada tarefa; desenvolve instrumentos de avaliação; cria um plano de execução do projeto; e desenvolve o conteúdo selecionando materiais e recursos, dando preferência à reutilização [Clark 2004].

Tabela 1 - Comparativo entre os modelos instrucionais.

\begin{tabular}{|c|c|c|c|c|c|}
\hline Modelos & \multicolumn{3}{|c|}{ Concepção } & \multicolumn{2}{|c|}{ Execução } \\
\hline ADDIE & Análise & Design & Desenvolvimento & Implementação & Avaliação \\
\hline ASSURE & Análise & Objetivos & $\begin{array}{c}\text { Selecionar Mídia e } \\
\text { Materiais }\end{array}$ & $\begin{array}{l}\text { Utilizar Mídia e } \\
\text { Materiais } \\
\text { Requisição do } \\
\text { Aprendiz } \\
\end{array}$ & $\begin{array}{l}\text { Avaliação e } \\
\text { Revisão }\end{array}$ \\
\hline PIE & \multicolumn{3}{|c|}{ Planejamento } & Implementação & Avaliação \\
\hline AIM-CID & Conceitual & Instrucional & Didática & & \\
\hline 4C/ID & Ativação & \multicolumn{2}{|c|}{ Demonstração } & Aplicação & Integração \\
\hline $\begin{array}{l}\text { ILDF On- } \\
\text { line }\end{array}$ & Exploração & \multicolumn{2}{|c|}{ Enactment } & \multicolumn{2}{|c|}{ Avaliação } \\
\hline DEI & $\begin{array}{l}\text { Identificação, } \\
\text { Caracterização e } \\
\text { Análise }\end{array}$ & $\begin{array}{l}\text { Objetivos } \\
\text { Seleção de } \\
\text { recursos }\end{array}$ & $\begin{array}{c}\text { Projeto de } \\
\text { estratégias de } \\
\text { ensino } \\
\text { Fluxo de atividades }\end{array}$ & Desenvolvimento & Avaliação \\
\hline \multirow{2}{*}{$\begin{array}{l}\text { Gerlach e } \\
\text { Ely }\end{array}$} & \multirow{2}{*}{$\begin{array}{l}\text { Identificação do } \\
\text { Conteúdo e dos } \\
\text { Objetivos }\end{array}$} & \multirow{2}{*}{\multicolumn{2}{|c|}{ Avaliação dos Conhecimentos Prévios }} & \multirow{2}{*}{ Desenvolvimento } & $\begin{array}{l}\text { Avaliação de } \\
\text { Desempenho }\end{array}$ \\
\hline & & & & & Feedback \\
\hline \multirow{3}{*}{$\begin{array}{l}\text { Dick e } \\
\text { Carey }\end{array}$} & $\begin{array}{c}\text { Definição dos } \\
\text { objetivos }\end{array}$ & $\begin{array}{c}\text { Estratégia de } \\
\text { aprendizagem }\end{array}$ & \multirow{3}{*}{$\begin{array}{l}\text { Conteúdos, } \\
\text { materiais e } \\
\text { recursos. }\end{array}$} & \multicolumn{2}{|c|}{ Pré-avaliação do DI } \\
\hline & Roteiro pedagógico & $\begin{array}{l}\text { Instrumento de } \\
\text { avaliação }\end{array}$ & & \multicolumn{2}{|c|}{ Avaliação contínua do DI } \\
\hline & $\begin{array}{c}\text { Nível de } \\
\text { conhecimento }\end{array}$ & $\begin{array}{c}\text { Plano de execução } \\
\text { do projeto }\end{array}$ & & \multicolumn{2}{|c|}{ Revisão do DI } \\
\hline
\end{tabular}

No modelo PIE a fase de planejamento define como as aulas serão apresentadas, a fase de implementação trabalha os métodos de ensino-aprendizagem e os meios de comunicação, e na fase de avaliação são avaliados a aprendizagem e os métodos de ensino apresentados [Gutierrez 2013]. No modelo ILDF Online a fase de exploração analisa e documenta todas as informações relevantes sobre o cenário instrucional, na fase de enactment é realizada uma ligação das informações obtidas na fase anterior com estratégias instrucionais apropriadas para atingir todos os envolvidos, e na fase de avaliação são determinados os propósitos, resultados desejados e métodos de avaliação.

O modelo 4C/ID contém quatro fases, que não se assemelham com as fases dos demais modelos. Na fase de ativação os alunos recordam suas experiências anteriores e são estimulados a receber novos conhecimentos. Já na fase de demonstração a informação é apresentada através de exemplos, mídias, áudios, gráficos, entre outros. $\mathrm{Na}$ fase de aplicação são oferecidas aos alunos múltiplas oportunidades de aplicar o que aprenderam, e na última fase, denominada integração, os conhecimentos adquiridos são integrados à vida pessoal do estudante [Merriënboer et al. 2002].

O modelo IAM - CID constitui-se de três etapas, que mais se aproximam com a etapa de concepção de Filatro (2008). Na etapa de modelagem conceitual define-se o nível de conhecimento para o ensino-aprendizagem, observando os conceitos, a inter- 
V Congresso Brasileiro de Informática na Educação (CBIE 2016)

Anais do XXVII Simpósio Brasileiro de Informática na Educação (SBIE 2016)

relação dos assuntos, e a estrutura dos elementos propostos para o ensinamento [Barbosa 2004]. Na etapa instrucional além de conceitos, podem ser inseridos vários elementos para o conhecimento e domínio do assunto. Na etapa de modelagem didática, o desenvolvimento parte do modelo instrucional, incluindo estratégias de utilização e explanação do conteúdo [Barbosa 2004].

O modelo DEI é composto por nove elementos que podem ser divididos em cinco etapas: na primeira etapa são identificados os problemas instrucionais, especificadas as metas para a concepção de instrução e examinadas as características dos alunos; na segunda etapa são identificados os conteúdos e analisados os componentes de tarefas relacionadas aos objetivos e finalidades expostos; na terceira etapa são especificados os objetivos instrucionais e a sequência do conteúdo dentro de cada unidade de ensino para a aprendizagem lógica; na quarta etapa são definidas as estratégias de ensino e selecionados os recursos para apoiar as atividades de instrução; e na quinta etapa são desenvolvidos os instrumentos de avaliação [Alvarado et al. 2012].

A partir das análises realizadas, pode-se constatar que os modelos ADDIE, ASSURE, Gerlach e Ely e DEI são os mais completos. Todos eles apresentam a análise das características do estudante. Porém, destes apenas o modelo ADDIE analisa mais profundamente essas especificidades, levando em consideração o contexto tecnológico e o contexto do estudante para a construção do DI. Outra característica importante desse modelo é a organização da avaliação, que ocorre não só ao final da sua implementação, mas em todas as suas fases de desenvolvimento. Observa-se que o modelo DEI tem sua maior preocupação na definição dos objetivos da aprendizagem, baseando assim os demais elementos que o compõem. [Alvarado et al. 2012]. Já o modelo ASSURE demostra maior atenção com relação à escolha da tecnologia mais adequada ou a combinação de tecnologias para o trabalho com os variados conhecimentos [Santos 2010]. O modelo Gerlach e Ely, é mais voltado à definição de estratégias de ensino.

A partir das análises desenvolvidas nesta seção, cinco modelos podem ser destacados como os mais completos: ADDIE, ASSURE, Gerlach e Ely, DEI e Dick e Carey. Isso se deve ao maior número de fases que contêm e por apresentarem características mais definidas. Sabendo que o objetivo deste estudo é escolher uma metodologia para a construção de um DI para aplicação no MV TCN5, que possui características de adaptação ao estilo cognitivo e expertise do estudante, os modelos ADDIE e ASSURE são os que mais se enquadram. Porém levando em consideração o maior detalhamento do processo de construção de um DI, e a maior preocupação com o processo pedagógico o modelo Dick e Carey se destaca. Com relação à aplicação do DI em um MV, pode-se afirmar que os modelos ADDIE, ASSURE, DEI e Dick e Carey têm mais a oferecer, pois apresentam uma atenção especial à escolha dos recursos a serem utilizados. Além disso, o cuidado em avaliar e revisar o Design Instrucional construído é mais abordado nos modelos ASSURE e Dick e Carey.

Considerando o que foi exposto, os modelos ADDIE e Dick e Carey apresentam maior adequação com o contexto do MV TCN5, portanto sendo utilizados para a construção de um modelo de DI híbrido a ser tomado como base na elaboração do Design para a aprendizagem de Redes de Computadores no ambiente tridimensional. $\mathrm{O}$ modelo Híbrido pode ser conferido na Tabela 2, que apresenta em suas duas primeiras colunas os modelos ADDIE e Dick e Carey. A terceira coluna representa as fases seguidas para o desenvolvimento da proposta de DI para a aprendizagem de Redes de Computadores no MV TCN5. 
V Congresso Brasileiro de Informática na Educação (CBIE 2016)

Anais do XXVII Simpósio Brasileiro de Informática na Educação (SBIE 2016)

Tabela 2 - Modelo Híbrido de Design Instrucional.

\begin{tabular}{|c|c|c|}
\hline ADDIE & Dick e Carey & DI Híbrido \\
\hline \multirow{3}{*}{$\begin{array}{l}\text { Análise: expertise, estilo } \\
\text { cognitivo, largura de banda, } \\
\text { experiência online; Recursos } \\
\text { e conteúdos. }\end{array}$} & Identificar objetivos instrucionais. & \multirow{3}{*}{$\begin{array}{l}\text { Adaptação dos Objetivos } \\
\text { instrucionais ao estilo cognitivo e } \\
\text { expertise do estudante e escolha de } \\
\text { conteúdos. }\end{array}$} \\
\hline & Escrever objetivos de desempenho. & \\
\hline & Identificar comportamentos de entrada. & \\
\hline \multirow{4}{*}{$\begin{array}{l}\text { Design: Estratégias (tipos de } \\
\text { recursos, método de } \\
\text { avaliação). }\end{array}$} & Realizar análises de instrução. & \multirow{4}{*}{$\begin{array}{c}\text { Definição do nível de expertise } \\
\text { dos recursos e escolha das formas } \\
\text { de avaliação. }\end{array}$} \\
\hline & $\begin{array}{c}\text { Desenvolver testes de critério } \\
\text { referenciado. }\end{array}$ & \\
\hline & $\begin{array}{c}\text { Desenvolver e selecionar material } \\
\text { instrucional. }\end{array}$ & \\
\hline & $\begin{array}{l}\text { Desenvolver avaliação informativa e } \\
\text { contínua. }\end{array}$ & \\
\hline $\begin{array}{c}\text { Desenvolvimento: construção } \\
\text { do programa e adaptação de } \\
\text { materiais. }\end{array}$ & Plano de execução do projeto. & $\begin{array}{c}\text { Estruturação dos recursos dentro } \\
\text { dos ambientes do mundo virtual } \\
\text { TCN5 de acordo com os estilos } \\
\text { cognitivos }\end{array}$ \\
\hline $\begin{array}{l}\text { Implementação: Utilização do } \\
\text { curso pelos estudantes. }\end{array}$ & & $\begin{array}{l}\text { Utilização do curso pelos } \\
\text { estudantes. }\end{array}$ \\
\hline \multirow[t]{2}{*}{ Avaliação: do curso e do DI. } & $\begin{array}{c}\text { Condução da avaliação informativa e } \\
\text { contínua. }\end{array}$ & \multirow{2}{*}{$\begin{array}{c}\text { Condução da avaliação do curso e } \\
\text { da aprendizagem; analise dos } \\
\text { resultados e revisão do DI. }\end{array}$} \\
\hline & Revisar instrução & \\
\hline
\end{tabular}

Primeiramente definiram-se os objetivos do curso, seguido da adaptação dos conteúdos aos níveis de expertise e da definição das formas de avaliação de acordo com os melhores desafios para cada estilo cognitivo. Na sequência, especificaram-se os roteiros pedagógicos dentro de cada ambiente do mundo virtual TCN5, que representam os estilos cognitivos (Reflexivo, Serialista, Holista e Divergente) e os níveis de expertise (Básico, Intermediário e Avançado).

\section{Conclusão}

Atualmente existem diversos modelos para auxiliar no desenvolvimento de Design Instrucional, fazendo-se necessário escolher o que melhor se adeque ao propósito de cada curso. Nesse sentido, este estudo realizou uma análise comparativo-qualitativa de nove metodologias de desenvolvimento de DI utilizadas na educação, com o intuito de eleger a mais adequada para a estruturação de um curso de Redes de Computadores no mundo virtual TCN5. As metodologias incluídas nesta análise foram: ADDIE, ASSURE, PIE, AIM-CID, 4C/ID, ILDF Online, DEI, Gerlach e Ely e Dick e Carey.

A partir das análises realizadas foi identificado que os modelos ADDIE e Dick e Carey apresentavam maior adequação ao contexto de aplicação do DI para o MV TCN5, sendo então utilizados como base para a construção de um DI Híbrido, exposto na Tabela 2. Essa adaptação será utilizada para a elaboração de um planejamento que abranja as características de estilo cognitivo e expertise do estudante, contempladas no ambiente tridimensional.

Como trabalhos futuros pretende-se ampliar esta pesquisa visando incluir outras metodologias de desenvolvimento instrucional, bem como estruturar o DI para a aprendizagem ubíqua da disciplina de Redes de Computadores dentro de um ambiente virtual imersivo. 
V Congresso Brasileiro de Informática na Educação (CBIE 2016)

Anais do XXVII Simpósio Brasileiro de Informática na Educação (SBIE 2016)

\section{Referências}

Aceituno, R. G. A. (2013) "Aplicação da metodologia AIM-CID no Conteúdo da Disciplina Sistemas Operacionais". Dissertação de Mestrado. Instituição de Ciências Matemáticas e de Computação, ICMC - USP. São Carlos, SP.

Alvarado, S.; Canales, A.; Cabrera, I.; Cepeda, D.; Palomares, R. (2012) "Comparing the ADDIE \& Kemp Model". In: The University of Texas at Brownsville, College of Education Department of Teaching Learning and Innovation.

Bandeira, D. (2009) "Materiais Didáticos”. Editora: IESDE Brasil S.A. 456 páginas.

Barbosa, E. F. (2004) "Uma Contribuição ao Processo de Desenvolvimento e Modelagem de Módulos Educacionais". Tese de Doutorado. Instituição de Ciências Matemáticas e de Computação, ICMC - USP. São Carlos, SP.

Branch, R. B. ( 2009) "Instructional Design: The ADDIE Approach". In: Proceedings of the Second Sussex Conference, 1977, Volume 722 de Lecture Notes in Mathematics, Editora Springer Science \& Business Media. 213 páginas.

Clark, D. (2014) "Por Design Instrucional Sistema e ADDIE?" Disponível em<http://goo.gl/v5V2sA > Acesso em 22 de Fevereiro, 2014.

Filatro, A. (2008) "Design Instrucional na Prática". Person Education do Brasil. 173 p.

Grabowski, S. (2003) "Teaching \& Media: A Systematic Approach, The Gerlach \& Ely Model". Dr. Rob Branch EDIT 6180.

Gutierrez, G. (2013) "Newby, Stepich, Lehman and Russell (PIE Model) and ADDIE". Disponível em <http://goo.gl/EQK5KY> Acesso em 13 de julho 2015.

Heidrich; R.; Medina, G.; Salce, F. A. P. (2007) "Recomendações Ergonômicas para Interfaces: Design Instrucional para Alfabetização de Crianças com Necessidades Especiais". In. XVIII Simpósio Brasileiro de Informática na Educação.

Herridge, G. (2015) "The Use of Traditional Instructional Systems Design Models for eLearning”. Disponível em <http://goo.gl/KJ8FQW> Acesso em 14 de maio de 2015.

Holden, J.; Westfall, P. C. (2010) “An Instructional Media Selection guide for distance learning - implications for bended learning featuring an introduction to virtual worlds". In: USDLA: United States Distance Learning Association. Second Edition.

Horvitz, B. N. (2016) "Dabbagh. B. Bannan-Ritland: On-line learning: concepts, strategies and application". Disponível em <http://goo.gl/pvNeyj $>$ Acesso em 22 de maio de 2016.

Mallmann, E. M. (2008) "Mediação Pedagógica em Educação a Distância: cartografia da performance docente no processo de elaboração de materiais didáticos". Tese de doutorado. Programa de Pós-graduação em Educação, UFSC.

Merriënboer, J.; Clark, R.; Croock, M. (2002) "Blueprints for Complex Learning: The 4C/ID-Model”. In: ETR\&D. Vol. 50. No. 2, p. 39-64.

Morrinson, G.; Ross, S.; Kemp, J. (2004) "Designing effective instruction". (4rd ed.). New York: John Wiley \& Sons, Inc.

Nascimento, A. C. A. A. (2006) "O design do curso on-line favorecendo a construção de uma comunidade de aprendizagem de futuros professores". In: Revista Novas Tecnologias na Educação - RENOTE. V. 4 No 1, Julho.

Nunes, I. D.; Schiel, U. (2011) "Design Instrucional e seu acompanhamento em tempo de execução utilizando Rede de Atividades". In: XXII Simpósio Brasileiro de Informática na Educação - XVII Workshop de Informática na Educação.

Wazlawick, R. (2009) "Metodologia de Pesquisa para Ciência da Computação". 1" Edição. Rio de Janeiro: Elsevier Editora Ltda. 184p. 